\title{
Le sujet asymptotique et la filiation dans certains écrits d'Abdelkébir Khatibi
}

\author{
François Paré \\ University of Waterloo
}

\begin{abstract}
Résumé
Dans les espaces francophones, postcoloniaux ou minoritaires, la première langue reste, chez bon nombre d'écrivains, le lieu d'un interdit, l'individu étant cette fracture ou cette indécision même par laquelle s'infiltre son mode d'être particulier. De nombreux textes poétiques et d'essais contemporains témoignent d'une subjectivité obsédée par l'empreinte de cette première langue, langue souvent domestiquée, expurgée et rompue par l'expérience coloniale et l'héritage des nationalismes. Dans cette étude, nous tentons de réfléchir à cette question par le biais d'une lecture de certains écrits d'Abdelkébir Khatibi sur la langue maternelle et l'expérience de la filiation. En effet, chez ce romancier et penseur marocain, l'écriture témoigne d'une rupture ritualisée avec la famille et surtout avec le souvenir d'une enfance tourmentée. Plus largement, de nombreux textes de Khatibi soulèvent la question cruciale du statut symbolique de la langue première dans l'espace colonial nord-africain. Nous verrons que celle-ci, obturée par les strates successives de la colonisation, devient l'objet d'une quête épistémologique qui non seulement traverse Amour bilingue, récit philosophique entièrement consacré à cette question, mais imprègne l'œuvre tout entière de Khatibi.
\end{abstract}

Mots-clés : Khatibi, Postcolonialisme, Subjectivité, Mémoire, Filiation, Langue. 


\section{Introduction}

Qu'elle soir réelle ou purement symbolique, qu'elle soit maintenue précieusement ou tragiquement perdue, la langue première, écrit Catherine Mavrikakis, n'est jamais oubliée. Elle reste la trace de l'intégrité même d'un sujet identitaire dès lors obsédé par la recherche de son origine en elle :

[t]el serait le mythe que l'on pourrait désigner comme constitutif de notre pensée de la langue. La langue maternelle se verrait dotée des pouvoirs de l'objet-ruine: elle se dresse dans l'horizon humain lorsque tout a été effacé ou perdu. Pourtant la littérature s'inscrit souvent en faux contre cette théorie d'une éternelle plénitude de la langue ». (Mavrikakis, 1989: 59)

$\mathrm{Si}$, pour cette chercheure québécoise, la langue première, ainsi privée de l'événement de sa naissance, est marquée dorénavant par l'ambiguïté, si elle n'est plus guère qu'un « objet-ruine », fragile et lacunaire, elle n'en reste pas moins également un mystère à élucider, car c'est en elle que se profilent certaines figures problématiques de l'identité. En contexte postcolonial, n'est-elle pas « la subsistance d'une singularité non réductible » (Glissant, 1990 : 204) qui unit le sujet à une communauté identitaire élargie dont il tire malgré tout sa légitimité ?

Dans les espaces francophones, postcoloniaux ou minoritaires, cette nostalgie de la première langue reste, chez bon nombre d'écrivains, le lieu d'un interdit, l'individu étant cette fracture ou cette indécision même par laquelle s'infiltre son mode d'être particulier: "[1]a surconscience linguistique qui affecte l'écrivain francophone - et qu'il partage avec d'autres minoritaires - 
l'installe encore davantage dans l'univers du relatif, de l'a-normatif. Ici, rien ne va de soi. La langue, pour lui, est sans cesse à (re)conquérir » (Gauvin, 2010 : 78). On peut donc se demander ce qu'il en est aujourd'hui de ce sujet dans l'empreinte de sa première langue, langue souvent domestiquée, expurgée et rompue par l'expérience coloniale et l'héritage des nationalismes.

Dans les pages qui suivent, nous tenterons d'avancer des réponses à cette question par le biais d'une lecture de certains écrits d'Abdelkébir Khatibi sur la langue maternelle et l'expérience de la filiation. En effet, chez ce romancier et penseur marocain, l'écriture témoigne d'une rupture ritualisée avec la famille et surtout avec le souvenir d'une enfance tourmentée. Plus largement, à la manière d'Assia Djebar, par exemple, de nombreux textes de Khatibi soulèvent la question cruciale du statut symbolique de la langue première dans l'espace colonial nord-africain. Nous verrons que celle-ci, obturée par les strates successives de la colonisation, devient l'objet d'une quête épistémologique qui non seulement traverse Amour bilingue, récit philosophique entièrement consacré à cette question, mais imprègne l'œuvre tout entière. La question de la langue maternelle s'y pose de façon singulièrement marquée, faisant ressortir d'anciennes et de nouvelles hiérarchies, et provoque chez l'écrivain une vision lyrique de l'enfance perdue.

\section{La modernité postcoloniale et la rupture de l'origine}

Dans La mémoire tatouée, son « autobiographie d'un décolonisé », Khatibi s'insurge, en effet, contre le double carcan familial et colonial : 
[j]e connaissais déjà le terrorisme des pères ; je vivais avec le mien comme dans un jeu d'ombres, chacun son rôle et Dieu pour tous. J'appris - comme il convient - les offices du respect et du commandement, le code de la famille à barbe. Plus grand, je conquis l'espace à petits pieds, mais je retombai dans la fatigue de l'évasion, les solutions impossibles et le retour vaincu (Khatibi, I, $2008: 21$ ).

Ainsi, dès les premières pages de ce récit autobiographique surgit le problème du fils et de la filiation rompue et s'instaure ce que Matt Reeck appelle une «poétique de l'orphelinité » (Poetics of the Orphan). L'évocation d'un retour possible au lieu problématique de la naissance ne pourra s'effectuer à l'avenir que dans l'exercice d'une langue mémorielle fracturée par la différence inaugurale, par le «terrorisme» du père et par l'absence. Sorti symboliquement d'une enfance répressive, le personnage khatibien cherche à convoquer autour de lui et en lui les ressources d'une mémoire plurielle par laquelle le sujet marginalisé peut trouver à se manifester. L'écriture témoigne alors d'une subjectivité résurgente, structurée par son appartenance complexe à la culture marocaine :

Prenons l'être arabe, et particulièrement l'être marocain. Et bien, l'être marocain est profondément habité par son passé anti-islamique, par l'islam, par la berbérité, par l'arabité, par l'occidentalité. L'essentiel, donc, est de ne pas oublier cette multiple identité qui compose cet être, et, d'autre part, il s'agit de penser l'unité possible de toutes ces composantes, mais unité non théologique qui laisse à chaque part sa part et à l'unité la plasticité d'inspirer l'ensemble (Khatibi, cité par Ben Jelloun, 1978).

Cette subjectivité trouble, en constant déplacement, serait ainsi désignée dans toute l'œuvre de Khatibi par sa dérivabilité. C'est d'ailleurs dans les machinations du texte littéraire qu'un sujet, toujours multiple, souvent horsnorme, viendrait à naître à lui-même et aux autres et pourrait intervenir dans 
1'histoire. La filiation ne serait jamais reconstituée. Elle serait plutôt «une séparation à figurer dans un ensemble mouvant» (Khatibi, I, 2008 : 45), une distance à arpenter. Tels seraient les personnages et leur énonciation dans l'œuvre de l'intellectuel marocain, à la fois hors de la mémoire et en mémoire.

Dans les littératures contemporaines, le sujet postcolonial ou minoritaire, évoluant assez souvent en marge de sa propre histoire, reste avant tout un voyageur, un migrant, un itinérant, structuré, selon Khatibi, par sa rencontre avec les autres et par son expérience déterminante de la différence : « [1]e voyage est encore suspendu, alors que le livre a fait son entrée par une transplantation symbolique reçue de l'étranger », écrit Khatibi dans sa lecture de Segalen. On y lit encore :

Le voyage est une promesse, comme tout ce qui fait rêver à une révélation de soi, sinon à une certaine transfiguration. [...] Ainsi, me précédant dans ce regard croisé comme un sillage initiatique à ma séparation, il m'aura toujours enseigné à le tolérer dès le premier regard. (Khatibi, III, 2008 : 147)

Minorisée, objet d'une substitution de son identité première, l'instance subjective est donc habitée, selon l'auteur de « Célébration de l'exote », par une symbolique de l'étrangeté fraternelle, de sorte que la mémoire de cette rencontre avec la différence est constitutive de sa capacité de penser et d'agir. Porté par une troublante nostalgie, chez Khatibi comme dans tant de textes de notre époque, le sujet mis en scène par la littérature n'est jamais que le produit d'une déhiscence, tant il est frappé dans la profondeur de ses désirs par le mystérieux tremblement du départ et de la migration. Agile, ce point asymptotique, s'éloignant de son origine, «continue à obéir à une force souple et élastique qui détend les pas au- 
delà d'eux-mêmes et de leur trace » (Khatibi, III, 2008: 148, souligné dans le texte).

Voilà donc que, pour Khatibi, l'instance subjective, issue en partie de l'héritage colonial, n'a de substance que le mouvement. Prenant la mesure de la distance qui l'habite, elle est amenée tout à coup à s'effacer, à se voir comme une absence agissante : «[m]aintenant, tu marches vers la ville. Nous allons te pousser, Disciple, et ce récit - nourri du désir angélique - te poussera aussi vers ton premier enterrement. Tu quittes une torture pour une torture plus cinglante, et à force de quitter et de quitter, à force d'être immanquablement quitté et déserté, tu seras broyé » (Khatibi, I, 2008 : 147). Cette émigration méthodique au cœur de soi est donc à la fois une joie et une souffrance.

Dans La mémoire tatouée, ouvrage fortement influencé par l'expérience mystique, Khatibi emprunte largement au soufisme ${ }^{11}$. Trace d'une absence qui le fonde, le personnage autofictif semble alors fissuré, disjoint, nomade, comme l'enfant embourbé dans son malaise identitaire dans les premiers chapitres du livre. Fruit sans doute d'une lecture de Frantz Fanon et d'Aimé Césaire, la critique du colonialisme forme donc la base d'un déplacement de l'identité dont toute l'œuvre de l'écrivain cherchera à rendre compte. La paralysie fascinée du voyageur nostalgique, décrite par Aimé Césaire dans le Cahier d'un retour au pays natal semblait répondre à des impératifs semblables : «Partir », écrivait Césaire. «Mon cœur bruissait de générosités empathiques. Partir... j’arriverais lisse et jeune dans ce pays mien et je dirais à ce pays dont le limon entre dans la

\footnotetext{
${ }^{11}$ Voir à ce sujet la thèse d'Abdelkader Amri, La blessure créatrice : littérature et mysticisme chez Meddeb et Khatibi, Université de Grenoble-Alpes, 2018, en ligne : theses.fr - Abdelkader Amri , La blessure créatrice : littérature et mysticisme chez Meddeb et Khatibi.
} 
composition de ma chair: "J'ai longtemps erré et je reviens vers la hideur désertée de vos plaies » (Césaire, $1956: 41$ ). Cette collocation différenciatrice, à la fois ontologique et purement circonstancielle, d'une instance subjective placée devant la souffrance de son passé, se produit le plus souvent sur fond de distanciation et de rupture, comme le pensait Michel Foucault dans Les mots et les choses:

cette mince surface de l'originaire qui longe toute notre existence et ne lui fait jamais défaut (pas même, surtout pas à l'instant de la mort où elle se découvre au contraire comme à nu) n'est pas l'immédiat d'une naissance ; elle est toute peuplée de ces médiations complexes qu'ont formées et déposées dans leur histoire propre le travail, la vie et le langage » (Foucault, $1966: 341-342$ ).

Telle est aussi la leçon, on s'en souviendra, du père algérien illettré dans le roman de Faïza Guène, Un homme, ça ne pleure pas. Peu importe ses filiations, chez Guène comme chez Khatibi, la figure paternelle se construit sur la rature toujours recommencée de l'origine. Dans le récit de Guène, le personnage de Mourad ne cesse d'évoquer la nécessité d'abord vitale de revenir là où tout a commencé, dans cette Algérie du père qui l'obsède et qu'il rejette aussitôt pourtant du revers de la main : «c'est toujours la même rengaine : personne ne repart jamais de zéro, pas même les Arabes qui l'ont pourtant inventé, comme disait le padre » (Guène, 2014: 260). Or, que veut donc dire pour l'écrivain cette privation du commencement?

La distance asymptotique du sujet, s'éloignant infiniment de son centre, faisant de ce rayonnement la substance de son action sur le monde, constitue ainsi l'enjeu primordial de l'œuvre de Khatibi. Dans cette forme excentrée de l'espace et du temps, le fils éperdu, dont on voit déjà le profil dans La mémoire tatouée, 
retrouve le courage d'agir et de construire sa différence. Par son retour aux lieux inauguraux, il cherche à subvertir la normalisation imposée par les courants hégémoniques à l'œuvre dans la société post-coloniale. La nostalgie est alors pour lui une forme de la révolte. Le retour, si impossible qu'il soit, est en effet une exogenèse, une seconde naissance à partir de constituants hétérogènes. Dans ses écrits autobiographiques, Khatibi évoque à maintes reprises un fort sentiment d'imposture : [o]n peut commencer par n'importe où et tout le reste est hasard, chaque fois le souvenir est à gagner ou à détruire, une fois pour toutes, dans une fraude inavouée » (Khatibi, I, 2008 : 107). Tout est décalage dans cet univers de la trace. Sur cette déhiscence reposent, chez Khatibi, la liberté conditionnelle de l'écrivain et de son personnage, leurs pratiques nomades, leurs possibilités d'intervention et d'action, et leur attachement aux conditions perverses de leur venue au monde dans le non-pouvoir. En réalité, le sujet, indécis et vacillant, ouvragé quotidiennement par le refus du passé et, paradoxalement, une intense nostalgie pour ce lieu inaugural dont il a dû se détacher, est issu de l'excentration même qui nourrit l'histoire récente. Bien plus, ce sujet de la modernité postcoloniale est le produit d'un long processus de «désidentification » que reprend Khatibi dans ses essais critiques au milieu des années 1980 :

[1]a désidentification (plus ou moins marquée) de l'image de soi et de l'autre, affaiblit la notion de transmission et d'héritage, qui se constitue, de génération en génération, par la ritualisation et la mémorisation du legs ancestral. La désidentification peut provoquer un processus de rupture des chaînons de transmission, comme c'est le cas lors d'une colonisation prolongée par un pays sur un autre (Khatibi, III, $2008: 328$ ).

Pour Khatibi, l'urgence de tracer les contours pluriels de l'identité, d'être à l'écoute de ses tensions et contradictions inhérentes, d'en relever les fictions de 
l'originaire, n'a jamais été aussi pressante, puisque les sociétés de la modernité postcoloniale se constituent justement à même ces inégalités, à même une subjectivité déterminée par la minorisation et nourrie par une scission d'abord culturelle, puis linguistique, pour enfin devenir une fracture ontologique. Depuis La mémoire tatouée, tout revient à la figure paternelle. Le sujet n'arrivera donc à parler d'une seule voix qu'au terme d'un « parricide symbolique » (Khatibi, III, 2008 : 118). Depuis cette rupture de l'enfance, il n'est jamais tout à fait ici, ni tout à fait ailleurs. Il ressemble à ces oscillateurs harmoniques élusifs que la mécanique quantique cherche à reproduire pour mettre fin à la distance et à ses aspérités.

\section{Le Livre du sang et la filiation interrompue}

On peut alors se demander, dans un deuxième temps, si c'est bien la notion de sujet postcolonial, en tant que figure de résistance, qui nous permet alors de penser autrement les marges de notre monde. Ou si, au contraire, ce sont les marges, illuminées par la littérature, qui font miroiter les ruines de subjectivités troubles, néanmoins capables de transformation et de rédemption. La subjectivation serait alors une empreinte ciselée par les mots, les sonorités et les paysages d'une langue première. C'est ce code, dont il n'aperçoit plus que le dégradé, qui pourrait conférer au sujet rompu une légitimité principielle :

Ce dont un immigré a soif, écrit Amin Maalouf dans Le dérèglement du monde, c'est d'abord de dignité. Et même, plus précisément de dignité culturelle. La religion en constitue un élément, et il est légitime que les croyants veuillent pratiquer leur culte dans la sérénité. Mais pour l'identité culturelle, la composante la plus irremplaçable est la langue (Maalouf, 2009 : 263). 
Une telle conception du sujet identitaire ne suppose-t-elle pas le maintien de la filiation ininterrompue avec l'origine ? Or, nous avons vu que, dans La mémoire tatouée, le personnage mis en scène par Khatibi renonce à la continuité de son histoire. Plus tard, alors qu'il s'intéressera au patrimoine linguistique qui est le sien, l'écrivain convoquera les éléments d'une filiation aussi riche que difficile. En effet, dans l'univers postcolonial, la langue première est marquée par la substitution et l'absence, dira Khatibi dans « La langue de l'Autre », un texte de 1999 :

[c]ette langue n'est pas une propriété ; c'est plutôt le lieu vide d'une identité qui se réincarne. Rien donc n'est assuré, donné ou accordé par avance, sans le risque d'une division active de soi. On est plutôt dans l'horizon d'une promesse, une possibilité productrice, un jeu avec le hasard et l'inconnu (Khatibi, III, 2008 : 119).

Le sujet - interrompu dans son histoire propre - ne pourra donc se transformer en «possibilité productrice» que s'il consent à explorer et à théoriser l'insuffisance fondamentale de sa langue première, sa sujétion à travers elle à un ordre hiérarchique du monde qui renvoie à sa marginalité radicale.

Pourquoi s'exprime-t-il avec tant de réticence, lui qui se sent forcé à une «pseudonymie » symbolique, une désappropriation de la filiation identitaire ? Pourquoi ne se reconnaît-il pas dans cette langue première qui, il n'y a pas si longtemps, représentait transparence et légitimité ? «Celui ou celle qui se jette contre la nuit sauvage de la langue a la vocation d'un fantôme, d'un revenant archaïque » (Khatibi, III, 2008 : 118). Dans des textes très connus, Lise Gauvin identifie de façon extraordinairement précise le malaise linguistique qui anime le sujet, tel qu'il se manifeste dans ces textes : 
La langue, pour lui, est sans cesse à (re)conquérir. Partagé entre la défense et l'illustration, il doit négocier son rapport avec la langue française, que celle-ci soit maternelle ou non. Surconscience, c'està-dire conscience de la langue comme lieu de réflexion privilégié, comme territoire imaginaire à la fois ouvert et contraint. Une telle notion renvoie à ce que cette situation particulière dans la langue peut avoir d'exacerbé et de fécond (Gauvin, $2010: 8$ ).

C'est cette extériorité foncière du code qui est, chez Khatibi, l'objet d'une négociation constante. Pour le sujet, la langue n'est donc pas porteuse d'une adéquation entre soi et soi. Elle signifie l'étrangeté au cœur du pays revisité. Elle renvoie dès lors à un sentiment de perte définitive, une détresse devant l'impossible reconstruction de sa transparence primordiale.

Ce deuil de l'origine peut-il être renversé ? Par quel geste de tendresse le sujet rompu pourra-t-il à nouveau se blottir dans le lexique de son enfance ? Tout est dit en une seule intimation : le sujet contemporain pour Khatibi est appelé à mettre en œuvre sa condition paradoxale. Du récit de l'enfance et de l'affranchissement au sortir de l'adolescence ressortira la nécessité absolue de ce paradoxe en tant qu'espace de liberté. En 1983, dans son célèbre récit-essai Amour bilingue, Khatibi se penche à nouveau sur cette question de l'irrésolu en convoquant de manière plus explicite la langue première comme le lieu même d'un déchirement, comme une véritable scénographie des doubles. « N'ai-je pas grandi, dans ma langue maternelle, comme un enfant adoptif », s'interroge le narrateur dès les premières pages. Naissance à la complexité, effacement douloureux du lieu commun, quête paradoxale de l'origine, nécessité de l'identité adoptive, tous ces termes permettent de resituer le sujet khatibien dans sa relation difficile avec l'identité de sa langue maternelle, lui qui se décrit alors comme son « premier survivant visible » (Khatibi, I, $2008: 219$ ). 
Dans l'œuvre de Khatibi, le refus de l'assujettissement ne s'exprime pas d'emblée par une démarche ouverte sur l'avenir. Au contraire, dès Le livre du sang, récit initiatique paru en 1979, le personnage est marqué par ce que Khatibi appelle « une effervescence nostalgique » (Khatibi, I, 2008 : 119) qui le pousse à ne concevoir son existence que comme une quête réparatrice tournée vers la récupération du passé. Cette recherche acharnée se présente avant tout comme une épreuve spirituelle, fortement empreinte de mysticisme, comme en témoignent les premières lignes du chapitre s'intitulant «L'asile des inconsolés » :

[e]nfant inoubliable, avance vers nous en souriant. Avance avec la même complicité secrète. Enlève tes sandales et assieds-toi là en croisant les jambes. Ce simple geste sera toujours paré pour nous de nonchalance souple, orgueilleuse. On dirait que la pesanteur de la terre n'obéit plus qu'aux pas enchantés du Bien-Aimé. Viens! Affranchis-nous dans le corps lumineux d'un Ange vivant ! (Khatibi, I, $2008: 119$ )

Dans ce passage, les dernières invocations, insistantes, permettent de lier la construction du sujet individuel à la destinée collective des pèlerins formés, comme lui, par l'exigence d'une transcendance radicale. Dans ce roman initiatique, Khatibi établit ce qui deviendra dans toute son œuvre une mise en scène de la filiation rompue qui ne s'accomplit pour tous les engagés que par le dénudement de l'origine et sa sublimation par la nature en quelque sorte mystifiante du langage.

Cette seconde naissance du sujet prend la forme d'une « Apparition » (avec majuscule) et survient au terme d'une expérience ritualisée de la rupture. Ainsi, 
dans Le livre du sang, tout l'intérêt du récit réside justement dans la nécessité absolue de revivre par le paradoxe de l'écriture les conditions douloureuses du passé. Le sujet n'advient donc que par la lecture exigeante d'une enfance lancinante dont il ne reste pour lui que des traces, des empreintes et des évocations. Résoudre cette négativité fondamentale deviendra chez lui une obsession. Dès ce moment, le personnage khatibien conçoit l'enfance sur le mode de la perte. Cette absence est pourtant sa seule voie vers l'intelligibilité. Toute démarche constitutive de soi revient alors pour le « figurant » non pas à tenter de revivre le passé tel qu'il a pu être, mais à devenir symboliquement la « sépulture errante " d'une forme première toujours déjà comprise comme une absence. Voici ce que dit le narrateur du Livre du sang en s'adressant à chacun des disciples rassemblés au seuil de la quête initiatique :

Te voici parmi nous, renonçant à ta vie antérieure. Mais pour qu'un tel simulacre arrive à l'anéantissement, il faut que le simple d'esprit se souvienne de son ensourcement prénatal, qu'il traverse, par d'imperceptibles voiles, le sang immatériel de tous ses pères et frères, de toutes ses mères et sœurs, et de tous les êtres angéliques giclant du désir de mort. Entre le ciel et la terre, il y a des signes pour ceux qui savent. (Khatibi, I, $2008: 123$ )

Dans le roman, nombreuses sont les références à une dialectique du bien et du mal inspirée de la tradition religieuse, notamment du récit abrahamique du fils sacrifié. Ce grand récit du père acquiert pour le narrateur une indéniable transparence : « l'égorgement du fils d'Abraham qui est MON NOM INCARNÉ, un formidable cri, soutenu de siècle en siècle, de millénaire en millénaire » (Khatibi, I, 2008 : 149). Comme on peut le voir et comme le constate Fatima Ahnouch dans son étude de cette œuvre, le récit tend à surpasser le temps de l'histoire. «Il s'agirait, vraisemblablement d'une mémoire anticipée dont la 
contribution involontaire à l'abolition des structures temporelles provoque une sorte d'interaction entre le passé et le présent; une dialectique au profit de laquelle œuvre le récit. La mémoire se place, par conséquent, sous le signe de la rupture » (Ahnouch, $2004: 15)^{12}$. Si, dans l'épisode du fils sacrifié, la quête de subjectivation épouse des formes lumineuses, elle s'étend néanmoins bien audelà des témoignages anciens qui ne sont que des émanations d'événements inauguraux à jamais perdus.

Or, justement, le cri du fils se fait encore entendre, son nom appartient à l'histoire présente. C'est bien lui : il est le sujet ! Chez Khatibi, il n'y aura plus de pleine identité que dans la maitrise du simulacre de la filiation rompue. Le narrateur s'entraînera avant tout à déchiffrer les signes qui encombrent sa conscience. Il aura pour tâche de mettre en ouvre une herméneutique qui correspond à sa secondarité fondamentale, sa secondarité première, si l'on peut parler ainsi. C'est pourquoi, dans Le livre du Sang, le Maître ne pourra guider les participants dans leur recherche. La responsabilité retombera plutôt sur le disciple dont le visage épuré et la solitude exemplaire représentent pour l'écrivain les conditions de disponibilité nécessaires au renouvellement du savoir.

Jalon important dans l'évolution de la pensée de Khatibi, Le livre du sang établit donc la nécessité du retour. L'harmonie souhaitée par l'écrivain ne survient qu'au terme d'un « ébranlement » des certitudes du monde adulte, alors que le sujet, jusque-là sans domicile fixe, trouve dans les conditions androgyniques de l'enfance l'harmonie nécessaire à la consécration de son identité : «[d]ans le cœur stigmatisé, il y a une incroyable promesse surnaturelle

\footnotetext{
${ }^{12}$ Sur le sacrifice abrahamique chez Abdelkébir Khatibi, voir également l'essai d'Olivia C. Harrison, Transcolonial Maghreb: Imagining Palestine in the Era of Decolonization (2015).
} 
pour ceux qui sont infiniment de cette terre-ci : plus on s'ensevelit, plus le corps s'élève dans l'élan de la Passion, lévitation de l'être qui intervient, par éclair, dans toute âme possédée » (Khatibi, I, 2008 : 189). Face à sa dépossession historique, dont il est depuis longtemps la cicatrice, le sujet khatibien devient une instance exacerbée par ce manque. Faisant écho aux Derviches de la tradition soufie, l'écrivain invoque le travail difficile du paradoxe qui transformera la fracture du sujet en une substitution parfaite de l'origine. Dans les dernières lignes du Livre du sang, Khatibi évoque d'ailleurs la posture hiératique du sage dont les jambes croisées et l'immuabilité apparente symbolisent l'avènement d'une humanité réconciliée non pas avec mais par le passé.

Il convient de noter que le narrateur est une instance déchirée, en constante tension avec elle-même et dans ses rapports avec le temps et l'espace. Fruit d'un passé dont il ne lui reste que des empreintes voilées, il est hors de lui en quelque sorte, incapable de se résoudre à l'unité et à la mesure du réel. Un déplacement vers la notion cruciale de simulacre est au centre de la pensée d'Abdelkébir Khatibi au moment où l'identité subjective lui parait être une terrible illusion. Le sujet n'est-il pas toujours de l'ordre de la simulation ? Ou, mieux encore, un éternel substitut, selon la figure du fils, produit d'une rupture paradigmatique de la filiation. Retenons donc pour l'instant l'ensemble de ces éléments narratifs qui forment la substance du paradoxe de la langue première (présentée ici comme une androgynie), tel qu'il se déploiera quatre années plus tard dans Amour bilingue, une œuvre beaucoup plus proche de notre propos. 


\section{Identité asymptotique et langue première}

Amour bilingue, roman publié en 1983, cherche à préciser sur le mode euphorique les liens troubles entre le sujet et ses langues. Le roman de Khatibi s'ouvre sur une scène de distanciation exacerbée pour le personnage de l'amant par la venue de la saison estivale : «[1]es étés arrivaient avec fougue. Le vent l'enivrait. Impatience de se jeter dans l'eau, de se purifier de ses hantises. Il s'abandonnait à la natation et, ce soir, il s'était éloigné vers la haute mer » (Khatibi, I, 2008 : 211). Cet éloignement progressif de la rive et le déclin du jour qui s'annonce à l'horizon sont les indices d'un récit initiatique qui conduira le personnage (le «récitant»), mis en place par l'écrivain, au seuil de ce qu'il appellera «sa propre amnésie » (Khatibi, I, 2008 : 215). Le roman prend alors forme autour de la rencontre subite et inexpliquée d'une femme dont le narrateur deviendra éperdument amoureux. C'est sur cette trame assez simple que se déploie pour lui la question de la double langue dont il fera l'emblème de son identité subjective.

Il est important de noter que, dans cette œuvre, les langues sont appelées à coexister de façon harmonieuse et égalitaire sans que ne jouent les vicissitudes du pouvoir et les dépossessions de l'histoire. Cependant, un décalage persiste, signe d'une différence dont le narrateur comprendra éventuellement la nécessité heuristique. C'est pourquoi, dans les premières pages du roman, un doute persistant 1'habite : «[1]ui-même un simulacre, toujours là où il n'était pas, s'effaçant dans ses traces ? Et la folie de la langue ne l'offrait-elle pas à la folie du corps ? L'intraduisible qu'il entretenait avec ce danger si évident : une fuite, un refus obstiné de la langue maternelle » (Khatibi, I, 2008 :220) ? Comme dans Le livre du sang, Khatibi situe le récit dans un jardin édénique qui évoque tout 
de même, pour le personnage, sa ville natale maintenant porteuse de recommencement. Cette conjoncture lui paraît particulièrement positive, puisqu'elle s'appuie encore une fois sur l'hybridité mouvante du paradoxe :

il entrevoyait avec joie cette possibilité, ce recommencement unifiant deux lieux et deux continents, topographie d'une passion restant debout devant ses fragiles élaborations. Elle admirait en lui ce désir, comme si, à lui seul, celui-ci les avait liés l'un à l'autre, chacun le dernier de l'autre, entre deux langues - à la fin de ce siècle ». (Khatibi, I, $2008: 215$ )

Il n'est guère nécessaire d'insister ici sur l'impression de liberté absolue qui s'empare alors du récitant, lui qui avait été paralysé jusque-là, nous dit le texte, par la peur du péché et les « souffrances de l'enfer imaginaire » (Khatibi, I, 2008 :

216). Cependant, cette libération ne sera qu'une illusion et il le sait bien.

Si le titre du roman suppose la coexistence à travers l'être aimé de deux langues paritaires, l'émergence de cette problématique, au moment où la femme aimée exprime quelque résistance devant l'enthousiasme de son amant, permet de situer le sujet khatibien dans ses liens douloureux avec sa langue première. Car, construite sur la perte et la nécessité du simulacre, l'identité subjective ne cesse de faire écho à une dissociation fondamentale, survenue « un jour », au sortir de l'enfance, entre la langue première et ses substituts :

[1]angue dont il eut, un jour, honte, sous le coup d'une atroce humiliation. Mais il ne se souvenait pas de quelque fait marquant qui eût provoqué une telle sensation. Il se considérait comme un décadent, le dernier de sa généalogie, une sorte de mutant d'une langue à l'autre. (Khatibi, I, 2008 : 220)

Quelle est cette insuffisance du sujet dans sa langue première ? 
Là se trouve chez Abdelkébir Khatibi le sens d'une quête initiatique qui, bien que tournée vers l'avenir, met néanmoins en œuvre l'impureté linguistique du sujet, c'est-à-dire son incapacité à assumer pleinement ses liens avec le code normatif de son origine : « [t]raduire l'impur dans le pur, la prostitution dans l'androgynie, était une aventure, qui exigeait d'être vécue sans aucune réserve. Il errait de pays en pays, de corps en corps, de langue en langue. Et je tombais, parfois, dans la détresse » (Khatibi, I, 2008 : 221). Dans ce passage important, l'énoncé se présente d'abord sur le mode théorique, dénoté par l'infinitif du verbe traduire. Il s'agit ici d'une exigence programmatique qui, loin de s'accomplir pleinement, s'ouvre plutôt sur une subjectivité fragile, inapte à transcender la mouvance du simulacre. Du même souffle, l'œuvre annonce, du moins sur le mode du rêve, la persistance d'une communauté paritaire des langues dont le sujet, divisé en lui-même, serait le bénéficiaire et le condensé. Pour arriver à cet équilibre harmonieux, il aura fallu conjurer la figure maternelle au cœur même de la langue première. Car ce sujet qui, aux premières pages du roman, s'éloignait des rives, ne savait pas qu'il serait amené à abroger le principe même de sa filiation : « [p]ar effacement, par désir d'effacement, j'aurai besoin (en aurais-je l'insouciance ?) de nier toute descendance, et de célébrer cette procession de mères, de femmes, de putains et d'hommes révélés en moi » (Khatibi, I, 2008 : 265). Au premier regard, l'œuvre de Khatibi est traversée par une pluralité rédemptrice dont le sujet, au sortir de son expérience généalogique, semble sortir indemne, réconforté par une vision égalitaire de la filiation.

Cependant, cette sublimation du « Mal » n'est rendue possible que par la mise en place d'une logique nihiliste, que décrivent extrêmement bien Jean- 
Michel Devésa et Alexandre Maujean dans un texte de la revue Afrique contemporaine :

la réalité des hommes et des sociétés n'est pas en effet pleine ni homogène ainsi que l'accrédite leur perception sensible et idéologique, mais « trouée » et travaillée en son sein par un principe et une force de négation, un «néant fondateur», qui se confond probablement avec le mouvement même du monde, de la matière et de la vie. (Devésa et Maujean, 2012 : 35)

C'est cette « force de négation » (ce « miroir brisé », dans les termes de ces deux chercheurs), qui structure justement les rapports tortueux entre le sujet et ses langues chez Khatibi. En fin de parcours, c'est bien la figure de la mère qui se trouve à faire obstacle, car sa surprésence dans la conscience du récitant ne permet pas d'accéder sur le plan symbolique à l'androgynie rêvée. Peut-être n'y a-t-il de liberté au sens khatibien que dans le déni douloureux de la langue maternelle. Accéder à la maturité du sujet, ce serait donc faire le pari de la substitution, de la secondarité.

Au-delà du déchirement, le sujet de la filiation sait qu'il sera transporté dans l'avenir par la force du simulacre. Le récit abrahamique est clair : le fils condamné a été sauvé de justesse ${ }^{13}$. Il aurait pu y laisser sa peau. Et ce moment paradigmatique, ce sursis au sortir de l'enfance, a signifié son accès à la culture seconde, à cette culture adoptive qui est si intimement la sienne malgré tout. Chez Khatibi, la modernité postcoloniale, tel qu'elle se profile à l'orée de ces propos théoriques autant que dans les ouvrages antérieurs, ne cesse de produire de la

\footnotetext{
${ }^{13}$ Voir les textes cruciaux de Khatibi sur le sacrifice dans ses essais sur l'islam : « [n]ous ne sommes pas sortis de cet état hypnotique d'indétermination dans le sacrifice et de sa violence fondatrice » (Khatibi, III, $2008: 80$ ).
} 
différence, de l'éloignement, de la renonciation, de sorte que la subjectivité, loin d'engendrer l'unité cherchée, s'abolit plutôt dans cette troublante asymptote qui est son mode d'être. 


\section{Bibliographie}

Ahnouch, Fatima, Abdelkébir Khatibi, la langue, la mémoire et le corps. L'articulation de l'imaginaire culturel, Paris, L'Harmatan, 2004.

Amri, Abdelkader, La blessure créatrice : littérature et mysticisme chez Meddeb et Khatibi, Université de Grenoble-Alpes, 2018, en ligne : theses.fr - Abdelkader Amri, La blessure créatrice : littérature et mysticisme chez Meddeb et Khatibi.

Ben Jelloun, Tahar, «Entretien avec Abdelkébir Khatibi II », Le Monde, 15 février 1978, en ligne : Entretien avec Abdelkébir Khatibi II. - " Je ne peux qu'être avec ceux qui veulent vraiment changer la vie et la mort des Français " (lemonde.fr).

Césaire, Aimé, Cahier d'un retour au pays natal, Paris, Présence africaine, 1956.

Devésa, Jean-Michel ; Maujean, Alexandre, «L'Afrique dans la littérature : un continent en son miroir. Introduction thématique », Afrique contemporaine, $\mathrm{n}^{\mathrm{o}}$ 241, 2012, pp. 29-42.

Foucault, Michel, Les mots et les choses, Paris, Gallimard, 1966.

Gauvin, Lise, «Entre rupture et affirmation: les manifestes francophones », Études littéraires africaines, $\mathrm{n}^{\mathrm{0}} 29,2010$, pp. 7-14.

Glissant, Édouard, Poétique de la relation, Paris, Gallimard, 1990.

Harrison, Olivia C., Transcolonial Maghreb: Imagining Palestine in the Era of Decolonization, Stanford, Stanford University Press, 2015.

Jabri, Vivienne, The Postcolonial Subject: Claiming Politics / Governing Others in Late Modernity, Londres, Routledge, 2013.

Khatibi, Abdelkébir, I-Romans et récits, Paris, La Découverte, 2008.

Khatibi, Abdelkébir, III - Essais, Paris, La Découverte, 2008. 
Laroussi, Foued, (dir.), Insularité, langue, mémoire, identité, Paris, L'Harmatan, 2017.

Legg, Stephen, «Beyond the European Province: Foucault and Postcolonialism », dans Jeremy W. Crampton et Stuart Elden (dir.), Space, Knowledge and Power: Foucault and Geography, Londres, Routledge, 2007, pp. 265-288.

Maalouf, Amin, Le dérèglement du monde, Paris, Le livre de Poche, 2009.

Mavrikakis, Catherine, «La traduction de la langue pure: formation de la littérature », Carrefours de la traduction, vol. 2, n 1, 1989, pp. 59-74.

Paré, François, La distance habitée, $2^{\mathrm{e}}$ édition, Ottawa, David, 2020.

Reeck, Matt, « The Poetics of the Orphan in Abdelkébir Khatibi's Early Works », Journal of French and Francophone Philosophy / Revue de la philosophie française et de langue française, vol. XXV, n 1, 2017, pp. 132-149. 